\title{
AVALIAÇÃO DA TÉCNICA PROJETIVA DE RORSCHACH EM PORTADORES DO HIV ${ }^{1}$
}

\author{
Luciana Nogueira Fioroni ${ }^{2}$ \\ Marco Antonio de Castro Figueiredo \\ FFCLRP - Universidade de São Paulo
}

\begin{abstract}
Resumo: Os modos de enfrentar as vicissitudes decorrentes da contaminação pelo HIV estão ligados à história de vida do portador e aos recursos psíquicos disponíveis, revelando características de personalidade. Através da Técnica Projetiva de Rorschach, foram investigados os processos afetivos de portadores do HIV/ Aids, objetivando construir um quadro representativo dos recursos emocionais para lidar com a infecção. Participaram do estudo 20 portadores do HIV divididos em dois grupos: subgrupo $01-10$ pessoas soropositivas sintomáticas; subgrupo $02-10$ pessoas soropositivas assintomáticas. Os protocolos foram cotados segundo normas estabelecidas para a população de Ribeirão Preto. Os principais resultados indicaram ameaça em relação à integridade da identidade, juntamente com o medo da morte e o isolamento social. Observou-se a presença marcante da angústia de contato e as vivências emocionais dos pacientes com AIDS mostraram características mais rígidas e mais regredidas do que as dos portadores assintomáticos.
\end{abstract}

Palavras-chave: AIDS; aspectos de personalidade; enfrentamento

\section{EVALUATION OF RORSCHACH'S PROJECTIVE TECHNIQUE WITH HIV CARRIERS}

Abstract: The different manners of facing vicissitudes brought by the HIV infection are linked to the person's life history and the available psychological resources, revealing personality characteristics. Using Rorschach's Projective Technique, the affective processes of HIV carriers and patients with AIDS were investigated, in order to obtain a representative picture of their emotional resources for dealing with the infection. Twenty HIV carriers took part on the study, and they were divided as it follows: group $01-10$ symptomatic serum-positive persons and group $02-10$ asymptomatic serum-positive persons. The Rorschach's protocols were rated according to the patterns for the population of Ribeirão Preto. The main results indicated identity integrity menace, associated with fear of death and social isolation, contact anguish and the emotional experiences of patients with AIDS showed more rigid and retrieved characteristics than the asymptomatic serum-positive patients' ones.

Key-words: AIDS; personality aspects; coping

A vulnerabilidade aos distúrbios emocionais decorrentes da infecção pelo HIV parece envolver uma série de aspectos, entre eles os recursos pessoais, sociais e familiares, a flexibilidade nas respostas e o nível de aceitação da própria condição (Lopes, 1993). O modo pelo qual a pessoa enfrenta as situações decorrentes da contaminação traz à tona conflitos e sentimentos já existentes, mas que pela característica trau-

\footnotetext{
${ }^{1}$ Artigo recebido para publicação em 07/03/03; aceito em 29/08/03. ${ }^{2}$ Endereço para correspondência: Luciana Nogueira Fioroni, Departamento de Psicologia e Educação, Faculdade de Filosofia, Ciências e Letras de Ribeirão Preto, USP, Av. Bandeirantes, 3900, Monte Alegre, Ribeirão Preto, SP, Cep 14040-901, E-mail: luciananf@hotmail.com
}

mática do evento, tais conflitos são exacerbados. Neste sentido, parece não existir uma psicopatologia específica para o HIV, visto que a capacidade de preservar funções psíquicas estaria ligada a uma organização prévia da personalidade. O modo pelo qual são ativadas as defesas frente à angústia que representa a Aids, decorre das condições gerais de desenvolvimento e de vida dos pacientes. Neste sentido, é de extrema importância que se conheça os processos afetivos envolvidos no enfrentamento da síndrome, para construção de possibilidades adequadas de suporte emocional a estas pessoas. 
Vive-se hoje um novo desafio, muito diferente do que havia no início da epidemia. A sobrevida à aids se estendeu, o que implicou não somente em lidar com a própria morte, mas principalmente com a vida. Instala-se, então, uma situação em que a pessoa tem que enfrentar a idéia de uma morte anunciada que não tem tempo certo para ocorrer, gerando uma situação que tende a se alongar cada vez mais.

A fim de se entender melhor as reações sociais à Aids, é necessário considerar as crenças e práticas que estruturam as relações sociais, as interações sexuais, as trocas de sangue e o uso de drogas injetáveis. Essas práticas e seus significados são construídos social e culturalmente. Desta forma, as circunstâncias que moldam a disseminação do HIV também irão moldar as respostas sociais, individuais, as formas de interpretação da doença, as reações frente aos portadores/pacientes, as medidas de prevenção e as políticas públicas de saúde (Parker, 2000).

Trabalhos anteriores (Carson, V.; Soeken, K. L.; Chanty, J. \& Terry, L.1990; Figueiredo, 2001; Leserman, J.; Perkins, D. O. \& Evans, D. L. 1992) têm apontado dificuldades tais como a incerteza diante da expectativa de vida, o desenvolvimento flutuante da doença, que imprime no paciente e em seus familiares uma grande instabilidade afetiva. O estilo de vida pouco tradicional, suscetível a estigmatização, além do contexto sócio-econômicocultural deste segmento da população também são fatores de limitação para o enfrentamento da doença. (Coverdale , J. H. \& Aruffo, J. F. 1992; Pinto, 1988; Tunnel,1991). Tais questões de cunho psicossocial requerem um novo rearranjo, como por exemplo, conviver com situações de depressão, perdas e isolamento.

O papel desempenhado pelas transformações decorrentes da infecção interfere na qualidade de vida destas pessoas, que têm que buscar novas formas de enfrentar e solucionar problemas presentes e futuros, implicando em mudanças e adaptações ao próprio contexto (De Boer, J. B.; Spranger, M. A. G.; Aaronson, N. K.; Lange, J. M. A. \& Vam Dam, F. S. A. M. 1996; Green, 1995; Holmes \& Shea, 1997; Lopes, M. S.; Figueiredo, M. A. C. \& Turato, E.R. 1997)

Uma das reações mais correntes entre as pessoas infectadas pelo HIV é a culpabilização (Dresler \& Boemer, 1993; Fioroni, 2000; Nichols, 1995;
Vieira \& Sherlock, 1997). Além disso, são colocadas frente a frente com a possibilidade do próprio fim, fazendo com que tenham que lidar com a morte, causando o afloramento da vulnerabilidade humana até então não percebida (Cohen \& Faiman, 1993). Outra conseqüência se relaciona às constantes infecções oportunistas provocadas pela deterioração do sistema imunológico, levando a repetidas internações e debilidade física, provocando perda da esperança e exaustão emocional (Ferreira, 1994). A pessoa infectada pelo HIV comumente passa por um processo de regressão e tem dificuldades de integrar a própria história e a auto-imagem, sentindo sua vida fragmentada (Lopes, 1993). Neste processo, lança mão de algumas defesas psíquicas, como a não identificação com a doença e suas implicações, pois isto pode protegê-lo dos sentimentos agressivos que dirige a si mesmo e ao mundo externo, ligada à insegurança e à possível destruição do seu corpo e da sua vida. De qualquer forma, é importante que o profissional leve em conta o momento pelo qual está passando o paciente e que muitas vezes tais defesas são necessárias à manutenção de um equilíbrio, mesmo que este seja escasso. A história de perdas que as pessoas soropositivas passam interfere em seu senso de identidade, fazendo surgir a necessidade de recuperação de estágios anteriores de desenvolvimento, enquanto enfrentam conflitos relativos à integridade psíquica versus dissociação. (Berenstein, 1995; Jue, 1994; Rabkin, J. G.; Kemien, R.; Katoff, L. \& Willians, J. B. W., 1993).

Considerando as questões psicossociais apontadas anteriormente e seus efeitos na vivência cotidiana do portador/paciente; considerando também a importância da adesão ao tratamento clínico, através da construção conjunta entre equipe e pacientes, de estratégias adequadas de enfrentamento do HIV/Aids, buscou-se identificar os aspectos de personalidade, construindo um quadro dos recursos internos para lidar com a infecção pelo HIV. Estes dados podem oferecer subsídios para o planejamento de intervenções sensíveis às reais necessidades deste segmento.

\section{Método}

O trabalho está assentado em uma perspectiva qualitativa de pesquisa através da descrição e aná- 
lise da dinâmica de personalidade de portadores do HIV e pacientes com aids, relacionadas às vicissitudes do contágio.

\section{Sujeitos}

Participaram do estudo 20 pessoas, organizadas em 2 subgrupos:

Subgrupo 1 - 10 pessoas soropositivas sintomáticas, ou seja, que já desenvolveram os sintomas que caracterizam o quadro de aids.

Subgrupo 2 - 10 pessoas soropositivas assintomáticas, ou seja, que não haviam desenvolvido a doença.

Os critérios utilizados para compor o grupo de sujeitos foram: ter realizado exame anti-HIV e conhecer seu resultado positivo; estar em acompanhamento médico; ausência de sintomatologia psiquiátrica ou neurológica que inviabilizasse a compreensão dos instrumentos de pesquisa; condições físicas que permitissem participar das sessões de aplicação dos instrumentos; aceitação expressa do paciente em participar.

\section{Coleta de Dados}

Foram enviados ao Comitê de Ética em Pesquisa do Hospital das Clínicas da Faculdade de Medicina de Ribeirão Preto - USP, o projeto de trabalho e modelo do termo de consentimento. O pedido foi analisado e aprovado de acordo com o Processo HCRP n ${ }^{\circ}$ 5487/97.

A coleta de dados teve início através de contatos estabelecidos nos locais de atendimento desta parcela da população: a Unidade Especial de Tratamento de Doenças Infecciosas (UETDI) do HC-RP, e o Grupo de Apoio a Pacientes com Aids (GAPA-RP).

\section{Instrumentos de Investigação}

Entrevista de anamnese: Foi construído um roteiro sintético sobre informações pessoais para caracterizar de forma adequada o grupo escolhido e complementar o trabalho de análise e discussão.

Aplicação da Técnica Projetiva de Rorschach: Aplicação, em local adequado e reservado, da Técnica Projetiva de Rorschach para se obter informações referentes ao funcionamento da personalidade. Este instrumento fornece, através da projeção do sujeito sobre o material, dados relativos à estrutura e dinâmica atual da personalidade. Desta forma, a técnica possibilita englobar aspectos que dizem respeito ao modo de captar a realidade e entrar em contato com ela, observar e elaborar experiências (Cunha, 1986). A aplicação deste instrumento visou obter uma amostra do comportamento do sujeito em uma situação estímulo; tal amostra pode fundamentar hipóteses interpretativas que permitam fazer inferências sobre o funcionamento da personalidade em geral (Traubenberg, 1998).

\section{Tratamento dos Dados}

Os protocolos foram cotados e analisados com base nas normas estabelecidas por Pasian (1998) para a população de Ribeirão Preto. A partir das cotações, os protocolos foram avaliados e comparados por 02 juízes, a fim de obter os índices de acordos e desacordos. Os pontos de desacordos encontrados foram levados a um terceiro juiz a fim de realizar nova cotação e desempate das avaliações anteriores.

\section{Resultados}

\section{Caracterização dos sujeitos}

A caracterização dos sujeitos é apresentada na Tabela 1.

Sobre o grupo de pacientes soropositivos sintomáticos, o nível de escolaridade encontrado foi baixo, com média de 5.4 anos de escolarização. A via de contaminação deste grupo foi predominantemente sexual. O tempo de contaminação variou de 6 meses até 7 anos, com média de 3,4 anos de soropositividade. Os sujeitos 2, 4 e 5 são ex-usuários de drogas, e o sujeito 7 foi contaminado pelo marido que é ex-usuário de droga, desta forma percebese a presença da droga no cenário de contágio do HIV, como via indireta ou direta de contaminação.

Em relação ao grupo de portadores soropositivos assintomáticos, a média de anos de escolarização foi maior do que no subgrupo 1 , com 8,3 anos. A via de contaminação foi, na totalidade dos casos, sexual, sendo $40 \%$ por contato homossexual. O tempo de contaminação variou de 4 meses até 7 anos, com média de 3,6 anos de soropositividade. 


\section{Análise dinâmica dos protocolos de Rorschach}

Em relação ao índice de acordo dos protocolos de Rorschach, foi calculado através da equação I.A. = (å total - å desacordos / å total) x 100, e obteve-se o seguinte resultado: I.A. $=91,24 \%$

A validade do I.A foi calculada através da diferença de proporções:

$$
\mathbf{Z p}=\mathbf{p}-\mathbf{q} / \text { Öp.q/n, onde } \mathbf{p}=\text { acordos e } \mathbf{1}-\mathbf{p}
$$

Tabela 01: Caracterização dos sujeitos.

\section{$=\mathbf{q}$ (desacordos)}

Os resultados para os subgrupos 01 e 02 foram: Zp1 = 62,22 e Zp2 = 74,54; sendo o nível de significância menor que .0001.

Todos os protocolos foram avaliados e sintetizados para cada sujeito e feita a síntese dinâmica dos resultados obtidos para cada um dos subgrupos que compõem a amostra.

\section{Subgrupo 1 - Pacientes com Aids}

\begin{tabular}{|c|c|c|c|c|c|c|}
\hline Sujeito & $\begin{array}{c}\text { Sexo/Comp. } \\
\text { sexual }\end{array}$ & Idade & Estado civil & Escolaridade & $\begin{array}{c}\text { Forma de } \\
\text { contágio }\end{array}$ & $\begin{array}{c}\text { Tempo de } \\
\text { contágio* }\end{array}$ \\
\hline $\mathbf{1}$ & Masc./heteros & 37 & Divorciado & $1^{\circ}$ grau comp. & via sexual & 5 anos \\
\hline $\mathbf{2}$ & Masc./heteros & 28 & Solteiro & $1^{\circ}$ grau inc. & via sexual // UDI & 2 anos \\
\hline $\mathbf{3}$ & Masc./heteros & 27 & Solteiro & $1^{\circ}$ grau inc. & via sexual & 5 anos \\
\hline $\mathbf{4}$ & Masc./homo. & 20 & Solteiro & $1^{\circ}$ grau inc. & via sexual & 4 anos \\
\hline $\mathbf{5}$ & Masc./heteros & 36 & Amasiado & $1^{\circ}$ grau inc & UDI & 6 anos \\
\hline $\mathbf{6}$ & Fem./heteros. & 25 & Solteiro & $1^{\circ}$ grau inc & via sexual & 2 anos \\
\hline $\mathbf{7}$ & Fem./heteros. & 31 & Casada & $1^{\circ}$ grau inc & via sexual & 7 anos \\
\hline $\mathbf{8}$ & Fem./heteros. & 32 & Casada & $1^{\circ}$ grau inc & via sexual & 1 ano \\
\hline $\mathbf{9}$ & Masc./homos & 31 & Solteiro & $1^{\circ}$ grau inc & via sexual & 1 ano $4 \mathrm{~m}$ \\
\hline $\mathbf{1 0}$ & Fem./heteros. & 57 & Viúva & $1^{\circ}$ grau inc & via sexual & 6 meses \\
\hline $\mathbf{1 1}$ & Masc./homos & 36 & Solteiro & $2^{\circ}$ grau compl. & via sexual & 1 ano \\
\hline $\mathbf{1 2}$ & Fem./heteros. & 21 & Viúva & $1^{\circ}$ grau comp. & via sexual & 7 anos \\
\hline $\mathbf{1 3}$ & Masc./heteros & 44 & Solteiro & $2^{\circ}$ grau comp!. & via sexual & 2 anos \\
\hline $\mathbf{1 4}$ & Masc./homos & 21 & Solteiro & $3^{\circ}$ grau inc. & via sexual & 2 anos \\
\hline $\mathbf{1 5}$ & Masc./homos & 30 & Solteiro & $1^{\circ}$ grau inc & via sexual & 4 meses \\
\hline $\mathbf{1 6}$ & Masc./homos & 30 & Solteiro & $2^{\circ}$ grau compl. & via sexual & 2 anos \\
\hline $\mathbf{1 7}$ & Fem./heteros. & 27 & Casada & $1^{\circ}$ grau inc & via sexual & 1 ano $6 \mathrm{~m}$ \\
\hline $\mathbf{1 8}$ & Fem./heteros. & 27 & Amasiada & $1^{\circ}$ grau inc & via sexual & 5 anos \\
\hline $\mathbf{1 9}$ & Fem./heteros. & 36 & Solteira & $1^{\circ}$ grau inc & via sexual & 5 anos \\
\hline $\mathbf{2 0}$ & Fem./heteros. & 21 & Solteira & $1^{\circ}$ grau inc & via sexual & 1 ano \\
\hline
\end{tabular}

*Data do diagnóstico positivo para o exame anti HIV , fornecida pelo próprio sujeito

Tabela 02: Principais índices dos protocolos de Rorschach para o grupo de sujeitos soropositivos sintomáticos

\begin{tabular}{|c|c|c|c|c|c|}
\hline Sujeitos & Modo de apreensão & F\% & $\mathrm{F}+\%$ & Respostas K & Respostas H \\
\hline 1 & $\underline{G}$ Gbl D (Dd) & $65 \% \uparrow$ & $53,8 \% \uparrow$ & $\mathrm{K} \uparrow=1(\mathrm{Ra}, \mathrm{p} . \mathrm{X})$ & $0(0 \%)$ \\
\hline 2 & Gbl $\underline{\mathrm{D}} \underline{\mathrm{Dd}} \underline{\mathrm{Dbl}}$ & $71,8 \% \uparrow$ & $52,2 \% \uparrow$ & $K-=1(p . I X)$ & $\mathrm{H}=1, \mathrm{Hd}=1 \downarrow$ \\
\hline 3 & (G) $\underline{D} \underline{D d}$ & $72,2 \% \uparrow$ & $34,6 \% \uparrow$ & $\mathrm{K}-=1(\mathrm{p} . \mathrm{IX})$ & $\mathrm{H}=1, \mathrm{Hd}=4 \downarrow$ \\
\hline 4 & $\mathrm{G}(\mathrm{D})$ & $50 \% \uparrow$ & $21,4 \%$ & Kat $=1(p . I I I)$ & $\mathrm{H}=1 \downarrow$ \\
\hline 5 & (G) D (Dd) $\underline{\mathrm{Dbl}}$ & $66,6 \% \uparrow$ & $37,5 \% \uparrow$ & $\mathrm{K}=0$ & $\mathrm{H}=0$ \\
\hline 6 & (G) $\underline{D}$ & $45,4 \% \uparrow$ & $60 \% \uparrow$ & $\mathrm{K} \downarrow=1$ (p.III) & $\mathrm{H}=1 \downarrow$ \\
\hline 7 & (G) Gbl $\underline{D} \mathrm{Dd}$ & $21 \% \downarrow$ & $100 \% \uparrow$ & $\mathrm{K} \uparrow=1$ (p.IX) & $(H)=1 \downarrow$ \\
\hline 8 & (G) $\underline{D}$ (Dd) & $43,4 \% \uparrow$ & $80 \% \uparrow$ & $K=0$ & $\mathrm{H}=0$ \\
\hline 9 & $\underline{G} \underline{\mathrm{D}}$ & $26,6 \% \downarrow$ & $100 \% \uparrow$ & $\mathrm{K} \uparrow=1(\mathrm{p} . \mathrm{III})$ & $\mathrm{H}=1 \downarrow$ \\
\hline 10 & $\underline{G}(\mathrm{D})(\mathrm{Dd})$ & $36,4 \%$ & $0 \% \downarrow$ & Kat $=2$ (p.III e VII $)$ & $\begin{array}{r}\mathrm{H}=2,(\mathrm{H})=1, \\
\mathrm{Hd}=1,(\mathrm{Hd})=1\end{array}$ \\
\hline
\end{tabular}

$\uparrow$ índice acima da norma // $\downarrow$ índice abaixo da norma // $\mathrm{K} \uparrow$ - extensão // Kat - atitude // $\mathrm{K}$ - associado a má forma 


\section{Aspectos Cognitivos}

Em relação à produtividade média, este grupo obteve 19,3 respostas, um pouco acima da norma (Pasian, 1998). Ressalta-se que o aumento do tempo de latência e do número de respostas na última prancha do teste foi significativo para a maioria dos sujeitos, indicando a existência de ansiedades ligadas às situações de separação e de rompimento de vínculos. A função cognitiva desse grupo apresenta, de forma geral, prejuízo na apreensão global do real (sujeitos 2,3,5,6,7,8), indicando dificuldade nos processos de abstração e de busca de integração dos perceptos; aliado ao aumento do Detalhe (D), revela também a preocupação e o apego ao aspecto concreto e prático. Tais dados parecem refletir o contexto que a pessoa com aids tem que lidar; as conseqüências da doença contribuem para que as preocupações estejam voltadas ao próprio corpo, ao cotidiano.

Quanto à função intelectual, sete sujeitos $(1,2,3,4,5,6,8)$ apresentaram $\mathrm{F} \%$ acima da norma, destes, apenas o sujeito 4 apresentou $\mathrm{F}+\%$ dentro do esperado. Estes índices tratam da base do funcionamento lógico e da acuidade perceptiva do indivíduo, fatores decisivos para avaliar a integridade de funções egóicas e do tipo de vínculo desenvolvido com a realidade (Traubenberg, 1998). Além disso, a resposta formal adequada $(\mathrm{F}+)$ revela a organização do princípio da realidade, correspondendo à nitidez da percepção e atenção. Neste primeiro grupo, encontraram-se dois índices (F\% e F+\%) elevados, o que revela capacidade de controle egóico, mas também certo distanciamento dos aspectos afetivos, podendo-se considerar estes juntamente com o aumento das respostas Detalhe (D).

\section{Aspectos Afetivos}

Quanto às formas vivenciais obtidas através da técnica projetiva de Rorschach, os dados, apresentados na tabela 3 , mostram que:

\footnotetext{
${ }^{3}$ Cálculo do TRI: relação da soma das respostas cinestésicas (K) com a soma das respostas cor - $\sum \mathrm{K} / \sum \mathrm{C}$

Cálculo da TL: relação das respostas de pequenas cinestesias (kan,kob,kp) com a soma das respostas E (estompage) - $\sum \mathrm{k}: \sum \mathrm{Ep}$
}

Tabela 03: Vivência Afetiva dos pacientes com aids ${ }^{3}$

\begin{tabular}{c|c|c|c}
\hline Sujeito & TRI & TL & 3 F \\
\hline 01 & E.D. & E.D. & I \\
02 & E.D. & E.P. & E \\
03 & E.D. & E.P. & Ambigual (tend.I) \\
04 & E.D. & I.D. & I \\
05 & E.P. & Coartado & Ambigual \\
06 & Coartativo & I.P. & Ambigual \\
07 & E.D. & I.D. & E \\
08 & E.P. & I.D. & E \\
09 & E.D. & I.D. & Ambigual (tend.I) \\
10 & E.D. & I.P. & I \\
\hline
\end{tabular}

TRI - Tipo Ressonância Íntima // TL - Tendência Latente // $3^{*} \mathrm{~F}$. Fórmula Complementar // E.D. - extratensivo dilatado // E.P. - extratensivo puro // I.D. - introversivo dilatado // I.P. - introversivo puro

Foi feito um estudo de análise de diferença de proporções, para se obter dados a respeito da significância das tendências encontradas. Quanto à diferença entre as vivências afetivas, pode-se observar uma proporção significativa de sujeitos extratensivos (.90>.10; $\mathrm{Zp}=8,42 ; \alpha<.001$ ) para o Tipo de Ressonância Íntima (TRI). Em relação à Tendência Latente (TL), apesar dos índices de introversidade serem mais freqüentes, não houve uma proporção estatisticamente significante para esta forma (.60>.40; Zp 1,33; NS). O TRI representa a atitude fundamental da personalidade para consigo mesma e com o meio, determina o caráter e o modo de percepção, refletindo as ligações funcionais profundas (Traubenberg, 1998). O índice TL revela a presença ou não da angústia, tendo um papel complementar ao TRI, na forma de um indicativo potencial em relação ao que está atualizado pela ressonância íntima.

Os sujeitos da amostra são predominantemente extratensivos e possuem um tipo de adaptação ao meio muito mais determinado pelos afetos do que pelo pensamento, maior dificuldade de reflexão e parecem menos estáveis do ponto de vista da motilidade e da afetividade. O contato afetivo tende a ser tipicamente mais intenso e mais frágil, marcado pela labilidade e instabilidade.

Em relação à Tendência Latente (TL) as respostas deste tipo revelam a presença ou não de angústia, que neste grupo parece existir principalmente nos sujeitos 02 e 08 ; a maioria das 
respostas deste tipo estão ligadas à prancha VI, portanto, à conflitos da ordem da sexualidade. Neste sentido os dados indicam a existência de posturas introversivas, marcadas principalmente pela presença do elemento kan (movimento animal), que poderia indicar tendências conflituosas em relação à vivência e expressão dos afetos nestes sujeitos.

O Índice de Dinamismo Interno (K), revelado pelas respostas que indicam movimento humano, é um dos pontos mais importantes da técnica. Este índice esteve no seu valor mínimo esperado, $\mathrm{K}=1$, em sete sujeitos, dos quais quatro produziram a resposta movimento na prancha III, onde é esperado pelo menos uma resposta movimento. De forma geral, as figuras humanas são indeterminadas quanto ao gênero. Há recusa cinestésica, isto é, o sujeito não percebe o elemento humano na prancha III, nos casos 1,3,5,7,8; sendo que os sujeitos 5 e 8 não perceberam o movimento humano em nenhuma das pranchas. Fica evidente também uma certa depreciação do modelo humano e a dificuldade de identificação. Apenas os sujeitos 1,4,e 9 projetaram movimentos de troca, de interação humana marcada, os demais são movimentos neutros, com poucos elementos pessoais ou são imagens desvitalizadas.
Os dados de $\mathrm{K}$ situados na produção mínima esperada, aliados ao $\mathrm{H} \%$ rebaixado, e a predominância do TRI extratensivo, indicam a dificuldade destes sujeitos em voltarem a atenção e o interesse para si mesmos, a possibilidade de conhecerem a existência de um mundo interno próprio, fazendo com que os interesses e os comportamentos estejam voltados para o mundo externo, caracterizando um tipo de contato afetivo mais superficial.

\section{Aspectos Adaptativos}

A estereotipia do pensamento aparece em $60 \%$ dos casos $(3,6,7,8,9,10)$, onde o índice A\% está elevado. Em relação à apreensão das normas sociais, revelada pelas respostas banais (Ban\%), é adequada em $60 \%$ dos casos, mas apesar da capacidade de conhecimento e entendimento destas normas, a possibilidade de generalização está prejudicada $(02,03,05,06,07,08)$.

\section{Subgrupo 2 - Sujeitos Soropositivos}

Assintomáticos

Tabela 04: Principais índices dos protocolos de Rorschach para o grupo de sujeitos soropositivos assintomáticos

\begin{tabular}{|c|c|c|c|c|c|}
\hline Sujeitos & Modo de apreensão & F\% & $\mathrm{F}+\%$ & Respostas $\mathrm{K}$ & Respostas H \\
\hline 11 & (G) D Dd Dbl & $76,3 \% \uparrow$ & $34,5 \% \uparrow$ & $K=0$ & $\hat{\mathrm{H}}=1, \mathrm{Hd}=2 \downarrow$ \\
\hline 12 & (G) $\underline{\mathrm{D}} \mathrm{Dd} \underline{\mathrm{Dbl}}$ & $58,8 \% \uparrow$ & $70 \% \uparrow$ & Kat $=1$ (p.III) & $\mathrm{H}=4,(\mathrm{H})=1, \mathrm{Hd}=3 \uparrow$ \\
\hline 13 & (G) $(\overline{\mathrm{D}}) \underline{\mathrm{Dd}} \overline{\mathrm{Dbl}}$ & $62,5 \% \uparrow$ & $60 \% \uparrow$ & $\mathbf{K} \uparrow=1(\mathrm{p} . \mathrm{III})$ & $\mathrm{H}=1, \mathrm{Hd}=2$ \\
\hline 14 & $\underline{G}(\mathrm{D}) \overline{\mathrm{D}} \mathrm{bl}$ & $20 \% \downarrow$ & $75 \% \uparrow$ & $K+=1$ (p.III) & $\mathrm{H}=2, \mathrm{Hd}=3 \uparrow$ \\
\hline 15 & $\underline{G}(\mathrm{D})$ & $30,7 \% \downarrow$ & $75 \% \uparrow$ & $K+=1$ (p.II) & $(H)=1 \downarrow$ \\
\hline 16 & $\underline{G}(\bar{D}) \mathrm{Dd} \underline{\mathrm{Dbl}}$ & $33,3 \%$ & $33,3 \% \uparrow$ & $K=0$ & $(\mathrm{H})=1 \downarrow$ \\
\hline 17 & (G) $\mathrm{D} \underline{\mathrm{Dd}}$ & $41,2 \% \uparrow$ & $51,7 \% \uparrow$ & $\mathrm{K} \uparrow=1$ (p.III) & $\mathrm{H}=2, \mathrm{Hd}=1 \downarrow$ \\
\hline 18 & (G) $\underline{D}(\overline{\mathrm{Dd}})$ & $35,3 \% \mathrm{n}$ & $83,3 \% \uparrow$ & $\begin{array}{l}\mathrm{K} \uparrow=1(\mathrm{p} . \mathrm{X}) \mathrm{e} \\
\mathrm{Kat}=2(\mathrm{p} . \mathrm{I}, \mathrm{III})\end{array}$ & $\mathrm{H}=2,(\mathrm{H})=1, \mathrm{Hd}=3 \uparrow$ \\
\hline 19 & (G) $\underline{D}$ (Dd) & $66,6 \% \uparrow$ & $72,7 \% \uparrow$ & $\mathrm{K} \downarrow=1$ (p.III) & $\mathrm{H}=1,(\mathrm{H})=1, \mathrm{Hd}=2 \uparrow$ \\
\hline 20 & $\mathrm{G} \underline{\mathrm{D}}(\mathrm{Dd})$ & $33,3 \%$ & $66,6 \% \uparrow$ & $\mathrm{K}-=2$ (p.X) & $\mathrm{H}=5, \mathrm{Hd}=1 \uparrow$ \\
\hline
\end{tabular}

\section{Aspectos Cognitivos}

A produtividade média dos sujeitos foi de 20,1 respostas. Aqui também se nota fenômeno semelhante ao ocorrido no subgrupo 1 , em que a quantidade de respostas na última prancha aumentou em relação às demais. Quanto ao tipo de apreensão, obteve-se o índice de respostas globais (G) rebaixado em $60 \%$ dos sujeitos $(11,12,13,17,18,19)$; destes, todos apresentaram associação com respostas de boa qualidade formal. O índice de respostas detalhe (D) esteve elevado em $50 \%$ da amostra $(11,12,18,19,20)$ e rebaixado em $40 \%(13,14,15,16)$. O recurso das respostas globais indica, tanto mecanismo cognitivo 
quanto atitude afetiva, que pode ser utilizada como mecanismo defensivo contra perturbações ou ansiedades causadas por situações estressantes (Traubenberg, 1998). Aqui tal recurso defensivo encontra-se prejudicado e o sujeito tende a reagir de forma mais automatizada, mais concreta. Apesar de haver dificuldade na busca de uma unidade, inclusive unidade corporal, estes sujeitos assintomáticos apresentaram menor dificuldade neste aspecto do que os sujeitos sintomáticos.

Em relação ao funcionamento do pensamento, os sujeitos 11,12,13,17 e 19 apresentaram F\% acima da norma, e todos tiveram o $\mathrm{F}+\%$ elevado. Estes dados indicam que a função pensamento está desenvolvida neste grupo, mas considerando o $\mathrm{F} \%$ acima da norma, as reações tendem a ser mais impessoais; estas tendências são semelhantes no subgrupo 1. Este índice também revela o funcionamento egóico e seu poder de regulação, que neste grupo parece ser intenso, fazendo com que o controle das pulsões seja, muitas vezes, rígido.

\section{Aspectos Afetivos}

As formas vivenciais obtidas através da técnica projetiva de Rorschach, apresentaram os seguintes dados,:

Tabela 05: Vivências Afetivas dos portadores assintomáticos ${ }^{4}$

\begin{tabular}{c|c|c|c}
\hline Sujeitos & TRI & TL & 3 Fórmula \\
\hline 11 & E.P. & I.D. & Ambigual \\
12 & E.D. & Coartativo & Ambigual \\
13 & E.D. & E.D. & I \\
14 & E.D. & I.D. & I \\
15 & E.D. & E.D. & Ambigual \\
16 & E.P. & I.D. & Ambigual \\
17 & E.D. & I.D. & Ambigual \\
18 & E.D. & I.D. & E \\
19 & E.D. & I.P. & I \\
20 & E.D. & I.D. & E \\
\hline TRI - Tipo de Ressonância Intima // TL - Tendência \\
Latente // 3 F. - Fórmula Complementar // E.D. - \\
extratensivo dilatado // E.P. - extratensivo puro // I.D. - \\
introversivo dilatado // I.P. - introversivo puro
\end{tabular}

${ }^{4}$ Cálculo do TRI: relação da soma das respostas cinestésicas (K) com a soma das respostas cor $-\Sigma \mathrm{K} / \Sigma \mathrm{C}$

Cálculo da TL: relação das respostas de pequenas cinestesias (kan,kob,kp) com a soma das respostas E (estompage) - $\sum \mathrm{k}: \sum \mathrm{Ep}$
Quanto à diferença entre as vivências afetivas dos sujeitos, pode-se observar que a totalidade de sujeitos é extratensiva $(1.00>0 ; \alpha<.001)$ para o Tipo de Ressonância Íntima (TRI). Em relação à Tendência Latente (TL), os índices de introversidade apresentaram uma proporção estatisticamente significante para a forma introversiva $(.70>.30 ; \mathrm{Zp}=2,85 ; \alpha<$ .001)

Os sujeitos são extratensivos dilatados no modo de sentir e expressar suas necessidades emocionais e tendem a possuir um componente de Introversidade. Neste sentido, as pequenas cinestesias presentes são predominantemente kan, que refletem espontaneidade, espírito lúdico e labilidade, aproximando-se muito mais das características de extroversidade do que de introversidade.

Em relação ao índice de dinamismo interno (K), dois sujeitos o apresentaram acima do valor mínimo 1, os demais tiveram $\mathrm{K}=1$, e os sujeitos 11 e 16 , tiveram $\mathrm{K}=0$. Obtivemos recusa cinestésica nos sujeitos $11,15,16$ e 20, indicando pobreza imaginativa, além de controle excessivo no plano objetivo e temor de projeção, revelando retraimento e inibição da personalidade. As figuras humanas sexualizadas apareceram em cinco casos $(12,14,17,18,20)$. Os movimentos de interação ou troca apareceram nos sujeitos 13,14,15,17,19 e 20.

\section{Aspectos Adaptativos}

A tendência geral apresentada revela a predominância da Extratensividade como modo de se relacionar socialmente, mostrando que há tentativa de buscar contato. Neste grupo, $60 \%$ das pessoas tem o índice $\mathrm{H} \%$ elevado, muito diferente do grupo de pacientes sintomáticos. São dados positivos, pois indicam a possibilidade de percepção e identificação com o outro, podendo favorecer os vínculos estabelecidos. De modo geral, neste grupo observou-se tendência a melhor adaptação social afetiva do que nos pacientes com aids. Levando-se em conta o fato de que neste grupo de pessoas portadoras assintomáticas, a doença ainda não se manifestou, não afetando concretamente a auto-imagem e o corpo, favorecendo então melhor organização interna no se relacionar socialmente. 


\section{Análise das Particularidades}

A seguir, os principais resultados da análise das particularidades da Técnica de Rorschach através da avaliação do material estímulo são: Algumas pranchas foram selecionadas (III,V,VI e X) em função de terem revelado dados importantes sobre a organização da personalidade, como a auto-imagem, a integridade egóica, a vivência da sexualidade e as reações frente à situações de separação e perdas. Foram pranchas nas quais os sujeitos, de modo geral, apresentaram produções mais significativas.

\section{Prancha III}

As produções revelam um clima emocional mais negativo do que positivo. O simbolismo desta prancha refere-se à necessidade da representação de si face ao outro, é a prancha dos tipos de relações humanas procuradas. Notamos que para o grupo de pacientes com aids, apenas três sujeitos $(04,06,09)$ conseguem produzir resposta de conteúdo humano em interação, os demais produziram respostas com conteúdos disfóricos, desvitalizados e que fazem referência ao sentimento de angústia corporal, prejuízo da auto-estima representado pelos personagens iguais à "caveira", "marionetes" e "macaco feio", dificultando uma autopercepção positiva e a busca de contato com os outros, já que percebem a si mesmos e aos outros de forma denegrida. A necessidade de representação de si face ao outro está prejudicada, é vivida como algo ameaçador, desfigurado, em muitos casos a relação procurada não é de contato, mas de evitação $(1,3,5,7,8)$. Isto nos leva a pensar sobre a atuação de mecanismos de defesa que podem fazer do isolamento uma situação que protege do abandono, previamente sentido e sofrido; mas que posteriormente pode trazer inúmeros prejuízos à vida emocional e social destas pessoas. Além disso, há a questão da identidade do paciente com aids, que muitas vezes é construída sobre imagens estereotipadas de doentes terminais.

Para o subgrupo de portadores assintomáticos, os sujeitos 12,13,17,18 e 20 conseguem projetar elementos que remetem à busca de contato e possibilidade de vinculação, apresentando uma auto-imagem mais preservada. A necessidade de representação de si face ao outro parece estar mais satisfeita. Nota-se que a problemática de identificação surge nos sujeitos 11,15 e 16 que não conseguem perceber o elemento humano que lhes é natural, revelando um processo de negação da própria imagem, e da imagem corporal também. Nos sujeitos 14 e 19 o aspecto humano está desvitalizado e mal definido, além de apontar para presença de conflitos sexuais.

\section{Prancha V}

Esta é a prancha mais estruturada, sendo o estímulo muito próximo da realidade objetiva; seu simbolismo relaciona-se à integridade psíquica e física. Os pacientes com aids $(3,4,5,6,9,10)$ produziram repostas esperadas (ban), mas apenas com elementos cognitivos, com restrição de material projetivo. Os sujeitos 1,2,7 e 8 parecem atingidos pelas transformações da enfermidade e pelo sofrimento que ela causa. A possibilidade do sentimento de integridade leva à noção de si, que para estes sujeitos é desvitalizada, desintegrada. O prejuízo na percepção do real parece ser conseqüência das limitações que envolvem a autopercepção.

Seis sujeitos $(12,13,14,15,17,20)$ portadores assintomáticos produziram respostas esperadas nesta prancha, havendo em dois casos $(11,16)$ presença de mecanismos de regressão, bastante comum em pessoas soropositivas. Apenas o sujeito 20 não conseguiu integrar e organizar o estímulo, revelando grande desorganização interna e dificuldade em abordar a realidade evidente, fruto de processos defensivos como a negação.

\section{Prancha VI}

De modo geral os sujeitos apresentaram sinais diretos e indiretos de angústia relacionada à vivência da própria sexualidade. Respostas sem forma definida, comentários, conteúdos desvitalizados ou fálicos; quanto à apreensão, foi em sua maioria $\mathrm{D}$ ou $\mathrm{Dd}$, nos casos de respostas globais estavam associadas à má qualidade formal. A reação emocional foi mais negativa do que positiva, com confusão e incerteza em formular uma imagem para o estímulo. Esta prancha é carregada de implicações sexuais e de dinamismo, revela através do aspecto passividade/atividade a 
problemática de castração do sujeito, através dos objetos que podem ser dinâmicos ou passivos. Os pacientes com aids produziram em sua maioria imagens desvitalizadas, animais estáticos, conteúdo anatômico sem muito dinamismo.

Todos os sujeitos portadores tiveram uma reação emocional marcada frente ao estímulo, que foi de recusa e negação para 11,12,17 e 18, de espanto e mal-estar para 13,16 e 17 , de agressividade para o 14 e de confusão para o 15. Apareceram também sinais de esforço em conter e direcionar os impulsos sexuais. Os sujeitos homossexuais revelaram muita dificuldade em estabelecer a própria identidade sexual e impulsos agressivos dirigidos a ela. Em geral há receio de entrar em contato com a própria sexualidade; lembra-se o fato de que todos estes sujeitos foram contaminados por contato sexual, e tal experiência parece estar associada a sentimentos de morte, ameaça e culpa. Há nas respostas, tentativa de negar ou reprimir os desejos de viver a sexualidade, como se tivesse sido transformada em assunto tabu, proibido, porque remeteria às escolhas que levaram à infecção pelo HIV.

\section{Prancha X}

Esta última prancha vem com estímulos bem dispersos e dificulta a busca de unidade; remete às situações de separação ou término de vínculos. Todos os sujeitos aumentaram o número de respostas ou o tempo de latência, indicando que há muitas reações frente à separação. Para o grupo 1, sete sujeitos $(1,2,3,6,7,8,9)$ demonstraram de alguma forma, ansiedade em relação ao término da aplicação, que representaria a interrupção do vínculo.

Os sujeitos 15,16,17 e 18 (subgrupo 2) parecem superar a ansiedade de separação de forma positiva, acompanhada pela permissão à satisfação que o estímulo provoca. Há aspectos de agressividade nos sujeitos 14 e 20, há ansiedade de separação, medo de abandono com tentativa de apressar a ruptura do vínculo em busca de alívio, como no caso do sujeito 19 .

\section{$\underline{\text { Discussão }}$}

Percebe-se, ao final do trabalho, a diversidade de condições em que estas pessoas se inscrevem, suas formas de construção de respostas e alternativas para conviver com o HIV e com as conseqüências da infecção.

Para os pacientes com aids, a principal dificuldade revelada pela técnica de Rorschach reside na integridade da identidade, ameaçada pelo medo da morte, pelo medo do preconceito, pelo sentimento de culpa que afeta a auto-estima e por conflitos sexuais. Neste sentido, a angústia de contato aparece exacerbada em muitos casos e pode justificar os índices rebaixados de $\mathrm{H} \%$, prejudicando a busca de laços afetivos e sociais. As figuras humanas estão sempre relacionadas a características pejorativas, como "caveira”, "macaco feio”, “esqueleto”, evidenciando uma tendência à "desumanização", como se os sujeitos perdessem aos poucos suas qualidades humanas. A ansiedade de separação ficou marcada entre as pessoas contaminadas pelo HIV, surgindo uma reação emocional ambivalente: alívio e angústia diante do término do vínculo com o pesquisador. Ressalta-se a presença da agressividade, através de mecanismos projetivos.

Os pacientes com aids apresentaram um pensamento mais concreto com dificuldades de percepção global da realidade. A vivência emocional indicou posturas extratensivas, marcadas por angústia de contato e impulsividade. Desta forma, pode-se pensar em um funcionamento egóico desgastante para o paciente, onde parece haver a necessidade de um controle racional sobre as próprias emoções, na tentativa de proteção frente a manifestação dos impulsos e desejos.

A partir do conhecimento da própria soropositividade, estas pessoas sofrem uma série de transformações que implicam em reformular valores, hábitos, relacionamentos e objetivos; gerando intensos conflitos que abalam o senso de integridade física e psíquica, e a possibilidade de definir a própria identidade. Neste sentido, a visão das pessoas não contaminadas pelo HIV sobre o doente de aids acaba confirmando os sentimentos de rejeição e abandono vivido pelos portadores. Há o aspecto de culpabilização pela própria contaminação, há a introjeção do estigma, mas há também a rejeição real e concreta do mundo externo, fechando assim uma cadeia de relações limitadoras. As fantasias das pessoas soropositivas são testadas e muitas vezes con- 
firmadas pelo mundo externo, dificultando o trabalho de dissolução desta cadeia.

Surgiram questões ligadas à sexualidade, à figura feminina e sua representação simbólica. Na prancha da sexualidade (p.VI) as produções não tiveram forma definida, houve uma reação emocional significativa, presença de comentários e exclamações. As respostas revelaram sinais de esforços no sentido de conter os impulsos sexuais, principalmente entre os sujeitos femininos. Sua problemática principal relaciona-se à ambivalência quanto à vivência da sexualidade, havendo grande desejo e sentimento de falta dos contatos sexuais e também medo e agressividade em relação a este contato, que é sentido como ameaçador na medida em que está associado à contaminação, à uma ameaça de morte. Os sujeitos homossexuais masculinos apresentaram angústias frente à vivência da própria homossexualidade e das trocas sexuais relacionadas à representação da figura materna, ora vista de forma fragmentada e indefinida, ora vista como figura de proteção. Para estes sujeitos, há uma indiferenciação da identidade sexual e uma agressividade disfarçada dirigida à própria sexualidade associada à forma de contágio pelo HIV. As outras problemáticas são em relação à própria identidade e integridade física sentidas como ameaçadas e desvitalizadas, uma necessidade intensa de vínculos e carência afetiva.

O medo de morrer, a sensação de abandono e a angústia ligada à definição da própria identidade são destacados, tanto através da técnica projetiva quanto nos relatos dos sujeitos. A importância de construir meios eficazes de intervenção a fim de minimizar tais perdas é mais uma vez verificada. Vários estudos sobre intervenções de suporte à pessoas com aids, confirmaram que a existência de redes de atendimento que minimizem as perdas sofridas em decorrência da doença, auxilia o desenvolvimento do melhor equilíbrio emocional e melhor resposta ao tratamento. (Figueiredo, M. A.C.; Costa, J. C.; Coelho,W.F.; Trindade, E. \& Siqueira, M. R. 1999; Jue, 1994; Nord, 1997; Rabkin J. G.; Kemien, R.; Katoff, L. \& Willians, J. B. W., 1993).

Os resultados encontrados podem servir de material para um necessário processo de reorientação de como abordar o portador, que leve em conta uma visão completa, o aparato psíquico e orgânico funci- onando em conjunto, não fragmentado pelas várias especialidades técnicas. Desta forma, o portador pode se tornar sujeito do próprio tratamento, tomando para si muitas das responsabilidades e iniciativas em relação à própria enfermidade. O investimento real nas potencialidades destas pessoas é importante por permitir que haja espaço para a expressão da subjetividade e possibilitar alcance de níveis maiores de independência e autodeterminação.

\section{Referências Bibliográficas}

Berenstein, I. (1995). Realite psychique et technique clinique. Revue Francaise de Psychanalyse, 59(1): 189-96.

Carson, V.; Soeken, K. L.; Chanty, J. \& Terry, L. (1990). Hope and spiritual self-being: Essencial for living with AIDS. Perspectives in Psychiatric Care, 26(2): 28-34.

Cohen, C. \& Faiman, C.J. (1993). AIDS: Ataque ao sistema de defesas psíquicas. Bioética, ConseIho Federal de Medicina, 1(1): 67-70.

Coverdale , J. H. \& Aruffo, J. F.(1992). AIDS and family planning counseling of psychiatrically ill women in community mental health clinics. Mental Health Journal, 28(1): 13-19.

Cunha, J.A. (1986). Psicodiagnóstico. Artes Médicas: Porto Alegre.

De Boer, J. B.; Spranger, M. A. G.; Aaronson, N. K.; Lange, J. M. A. \& Vam Dam, F. S. A. M. (1996). A study of the reliability, validity and responsivesness of the HIV. Overview of problems evaluation system (HOPES) in assessing the quality of life patients with aids and symptomatic HIV infection. Netherlands. Quality of Life Research - An International Journal of Quality of Life Aspects of Treatment, Care and Rehabilitation, 5(3): 339-47.

Dresler, D.E. \& Boemer, M.R. (1993). O ser com AIDS em sua dimensão existencial. Revista Brasileira de Enfermagem, 46(1): 7-20.

Ferreira, C.V.L. (1994). AIDS e Vida: um estudo clínico-psicanalítico com pacientes HIV. São Paulo: Editora Lemos.

Figueiredo, M. A.C.; Costa, J. C.; Coelho,W.F.; Trin- 
dade, E. \& Siqueira, M. R. (1999). Grupos de suporte a pacientes com aids na Unidade Especial de Terapia de Doenças Infecto-contagiosas do Hospital das Clínicas da FMRP. Instituto de Estudos Avançados- USP, 13(35):157-59.

Figueiredo, M.A.C. (2001). Algumas questões psicossociais do atendimento a pessoas que convivem com HIV/Aids, sob o ponto de vista de um grupo de profissionais de saúde. Jornal Brasileiro de Aids, 2(1):17-24.

Fioroni, L.N. (2000). Modos de enfrentamento da aids e condições de vida. Estudo baseado em aspectos de personalidade e hierarquia de necessidades. Dissertação de Mestrado apresentada ao Departamento de Neurologia, Psiquiatria e Psicologia Médica da FMRP-USP.

Green, G. (1995). Aids and euthanasia. AIDS Care,7(suppl.2): 169-173.

Holmes, W. \& Shea, J. (1997). Performance of a new HIV/AIDS targeted quality of life (HATQOL) instrument in asymptomatic seropositive individuals. Quality of Life Research - An International Journal of Quality of Life Aspects of Treatment, Care and Rehabilitation, 6(6): 556-71.

Jue, S. (1994). Psychosocial issues of aids long-term survivors. Special issue: HIV/AIDS. Aids Project Los Angeles, Families in Society, 75(6): 324-32.

Leserman, J.; Perkins, D. O. \& Evans, D. L. (1992). Coping with de threat of AIDS. The role of social support. American Journal Psychiatric, 149(11): 1514-20.

Lopes, M.S. (1993). Estudo Qualitativo de Características Psicossociais de Pacientes Contaminados pelo HIV. Dissertação de Mestrado, Universidade Estadual de Campinas - Faculdade de Ciências Médicas, Campinas, 210p.

Lopes, M. S.; Figueiredo, M. A. C. \& Turato, E.R. (1997). Psichosocial characteristics of patientes infected by HIV and adherence to treatment in a Public Service in Brazil. Trabalho apresentado no $14^{\text {th }}$ World Congress on Psychosomatic Medicine. Sidney, Austrália, s/p.
Nichols, S.E. (1995). Psycho-social reactions of persons with adquirid immunodeficiency syndrome. Annals of Internal Medicine, 103, 756-57.

Nord, D. (1997). Threats to identity insurvivors of multiple AIDS-related losses. Seatlle AIDS support Group, American Journal of Psychoterapy, 51(3):387-402.

Parker, R. (2000). Na contramão da AIDS. Sexualidade, intervenção, política. Rio de Janeiro: ABIA; São Paulo: Editora 34.

Pasian, S.R. (1998). O Psicodiagnóstico de Rorschach: Um Estudo Normativo em Adultos na Região de Ribeirão Preto (SP). Tese de Doutoramento, FMRP-USP, Programa de PósGraduação em Saúde Mental, v.1 e 2, Ribeirão Preto.

Pinto, W. (1988). Como fica o atendimento? Jornal da ADUSP, São Paulo, p.12.

Rabkin, J. G.; Kemien, R.; Katoff, L. \& Willians, J. B. W., 1993). Resilience in adversity among long-term survivors of aids. Hospital and Community Psychiatric, 44(2): 162-67.

Traubenberg, N.R. (1998). A Prática do Rorschach. São Paulo: Vetor Editora Psico-pedagógica Ltda.

Tunnel, G. (1991). Complications in group psychoterapy with AIDS patients. International Journal Group Psychoterapy, 41(4): 481-497.

Vieira, N.F.C. \& Sherlock, M.S.M. (1997). O (des)velamento do cotidiano do indivíduo soropositivo. Jornal Brasileiro de Doenças Sexualmente Transmissíveis, 9(4): 4-7.

Apoio financeiro CAPES - trabalho derivado da dissertação de Mestrado de Renata Nogueira Fioroni, no PPG em Saúde Mental, da FMRP-USP. 